\title{
Vaskuläre Demenz und Hypertonie
}

\author{
Stephan Lüders, Joachim Schrader
}

Dementielle Erkrankungen sind eine zunehmende Belastung für das Gesundheitssystem. Da es bislang keine effektive Therapie gibt, ist die Primärpravention von herausragender Bedeutung. Zu den gesicherten Risikofaktoren für vaskuläre Demenzen, und vermutlich auch für Alzheimer, gehört die arterielle Hypertonie. Eine rechtzeitige medikamentöse antihypertensive Therapie kann kognitive Funktionseinbußen zumindest verlangsamen und eine Demenz hinauszögern.

\section{Definitionen}

Leichte kognitive Störungen (ICD-10 F06.7) [1]। Bei Weitem nicht alle kognitiven Einschränkungen sind mit einer Demenz gleichzusetzen [2]. Im Gegensatz zur Demenz beeinträchtigen leichte kognitive Störungen den Alltag der Patienten nicht oder nur in geringem Maß. Sie können allerdings ein Vorstadium einer Demenzerkrankung sein.

Demenz (ICD-10 F00-F03) [1] I Laut ICD-10-GM Version 2015 ist eine Demenz „ein Syndrom als Folge einer meist chronischen oder fortschreitenden Krankheit des Gehirns“ [1]. Bei einer Demenz sind viele höhere kortikale Funktionen gestört, das Bewusstsein ist jedoch nicht getrübt. Begleitet werden die kognitiven Beeinträchtigungen gewöhnlich von Veränderungen der emotionalen Kontrolle, des Sozialverhaltens oder der Motivation. Diese Symptome können auch früher eintreten.

Der Begriff Demenz beschreibt eine heterogene Gruppe von Erkrankungen unterschiedlicher pathophysiologischer Mechanismen mit dem Leitsymptom „kognitive Störung“ [3].

Vaskuläre Demenz (VD) | Zur Diagnose einer VD ist es erforderlich, vaskuläre Hirnveränderungen im CT oder MRT nachzuweisen [4]. Bei Patienten mit VD sind höhere kortikale Funktionen wie Schreiben, Lesen und Rechnen länger erhalten als bei Patienten mit Alzheimer-Demenz (AD). Bei VD finden sich 3 größere Gruppen:

1. Defektsyndrom nach größeren, einzelnen oder multiplen, ischämischen oder hämorrhagischen Insulten (Symptomatik sehr variabel).

2. hypertensive zerebrale Mikroangiopathie (multiple Lakunen der tiefen Kerne, diffuse Marklagerschädigung).

3. andere Formen bei Stenose oder Verschluss großer Gefäße und kleiner Gefäße (z. B. Grenzzoneninfarkte).

Bei mindestens 30\% aller Patienten finden sich „Mischdemenzen“, bei denen sich vaskuläre und degenerative Schädigungen überlagern [5].

\section{Diagnostik}

Sorgfaltspflicht | Für den betroffenen Menschen, sein familiäres, soziales oder berufliches Umfeld kann die Diagnose „Demenz“ weitreichende Folgen haben. Neben Angst vor Stigmatisierung oder dauerhafter Abhängigkeit sind auch depressive Veränderungen bis hin zu Suiziden bekannt. Im klinischen Alltag wird die Diagnose jedoch teilweise unkritisch benutzt, ohne dass empfohlene Kriterien der Diagnostik und Verlaufsbeobachtung eingehalten werden.

Pseudodemenz abgrenzen | Auch in Kliniken werden Patienten mitunter vorschnell als „dement“ klassifiziert - nicht nur, weil sich hieraus ggf. eine bessere Darstellung im DRG-System ergibt. Dabei wird vernachlässigt, dass eine Vielzahl von Akuterkrankungen oder Dekompensationen die kognitiven Funktionen z.T. drastisch verschlechtern können, ohne dass die Kriterien der Demenz erfüllt sind. Pathologische Ergebnisse in Tests, die zur Objektivierung beitragen sollen, sind daher häufig zu finden. Auf eine ambulante Kontrolle nach vollständiger Rekompensation wird oft verzichtet, so daß die Diagnose „Demenz“ bestehen bleibt, auch wenn es sich nur um eine Pseudodemenz bei Exsikkose, Stoffwechselentgleisungen, Infektionskrankheiten, unter bestimmten Medikamenten oder um ein Delir gehandelt hat.

Wichtig ist darüber hinaus, eine Depression oder Parkinson-Symptomatik abzugrenzen, die beide gerade bei älteren Patienten auftreten können [6, 7].

Umfassende Diagnostik | Aufgrund der Vielfalt an zugrundeliegenden Störungen ist eine sorgfältige Diagnostik entscheidend. Eine alleinige Diagnose durch „Biomarker“ ist bis heute nicht möglich [8, 9]. Die Diagnostik sollte sich z.B. an den S3-Leitlinien der DGPPN/DGN orientieren [10]:

- Quantifizierung der kognitiven Leistungseinbußen: z.B. MMST, DemTect, TFDD und Uhrentest [11-14] 
- Anamnese: kardio- und zerebrovaskuläre Erkrankungen, metabolische Risikofaktoren, Medikamente, körperliche Aktivität, früheres kognitives Leistungsniveau (Fremdanamnese)

- kardiovaskulärer und neurologischer Status

- EKG: zum Ausschluss von Vorhofflimmern

- laborchemische Basisdiagnostik

- Blutdruckmessung: Selbstmessung und 24hBlutdruckmessung (ABDM) obligat [15-17]

$\checkmark$ ggf. Liquoruntersuchung

- ggf. CT oder MRT des Schädels zur Differenzialdiagnostik bei bestehendem Demenzsyndrom

\section{Prävention}

Demenzrisiko | Risikofaktoren für einen Schlaganfall sind auch Risikofaktoren für die Entwicklung einer VD [18]. Kausale Assoziationen für arterielle Hypertonie im mittleren Lebensalter, Diabetes mellitus zu jeglichem Zeitpunkt und geringem Bildungsniveau im frühen Lebensalter sind gut belegt [1]. Je mehr Risikofaktoren vorliegen, desto größer ist auch die Wahrscheinlichkeit für kognitive Funktionsstörungen [19]. Mit dem Demenzrisiko assoziiert sind auch

- Vorhofflimmern,

- Herzinsuffizienz,

- koronare Herzkrankheit,

- Makroangiopathie,

- Alter und

- männliches Geschlecht.

Möglicherweise mindern vaskuläre Schäden die Reservekapazität des Gehirns und senken somit auch die Schwelle für die Manifestierung einer AD.

Das Demenz-Risiko kann reduziert werden durch eine

- bessere Kontrolle des Blutdrucks und weiterer kardiovaskulärer Risikofaktoren,

- optimale Einstellung eines Diabetes,

- Reduktion des Nikotinkonsums.

Ambulantes BlutdruckMonitoring

DemTect

Demenz-Detektion

(Screeningverfahren)

DGN

Deutsche Gesellschaft

für Neurologie

DGPPN

Deutsche Gesellschaft

für Psychiatrie und

Psychotherapie,

Psychosomatik und

Nervenheilkunde

MMST

Mini-Mental-Status-Test TFDD

Test zur Früherkennung von Demenzen mit Depressionsabgrenzung

Sport | Vermehrte körperliche Aktivität ist vermutlich ein wesentlicher Schlüssel, um diese Ziele zu erreichen [20]. Auch bei bestehenden kognitiven Einschränkungen zeigen sich positive Effekte. Dies geht aus einer Analyse von 14 Studien mit 1056 Patienten mit kognitiven Einschränkungen oderADhervor.DieTrainingsprogramme (2-4× pro Woche, jeweils 30-60 Minuten) enthielten

- Laufen,

- unterschiedlich intensives aerobes Training,

- Flexibilitäts- und Balance-Training,

- Krafttraining oder

- Tai Chi.

Die Patienten der Kontrollgruppen waren körperlich weitgehend inaktiv. Sie betrieben teils Kartenspiele, Handarbeiten und Dehnübungen. Körperliche Aktivität war mit einer signifikanten fektive therapeutische Intervention für Patienten mit kognitiven Defiziten oder Demenz [21].

Auch das Aufrechterhalten sozialer Kontakte trägt nachweislich dazu bei, das

Demenz-Risiko zu senken.

Geistige Aktivität I Die klinischen Auswirkungen einer beginnenden Demenz lassen sich z.T. durch kognitives Training oder Ausbildung vermindern [22]. Ein hohes Bildungsniveau bietet jedoch keineswegs einen zuverlässigen Schutz vor Demenz, wie sich an tragischen Einzelkasuistiken teils prominenter Personen erkennen lässt (z.B. Walter Jens, Ernst Albrecht, Margaret Thatcher).

\section{Stellenwert der Hypertonie}

Risiko zerebrale Ischämie | Die arterielle Hypertonie ist der wichtigste Risikofaktor für zerebrale Ischämien. Patienten mit Schlaganfällen haben wiederum ein stark erhöhtes Risiko für dementielle Erkrankungen. Die Häufigkeit wird mit 7\% im ersten Jahr bis zu $48 \%$ nach 25 Jahren berichtet [23-25]. Gegenüber klinisch manifesten Ischämien ist die Prävalenz von zunächst klinisch stummen Ischämien etwa 5-fach höher. Sie beträgt je nach Untersuchungsverfahren (cCT, MRT) 5-53\%.

- Schon bei bis zu 10\% der unter 50-jährigen Hypertonikern sind entsprechende Veränderungen zu finden.

- In einer Studie konnten bei 40,9\% der Patienten im Alter zwischen 50 und 60 Jahren Ischämien nachgewiesen werden, ohne dass bislang klinische Korrelate beobachtet worden waren [26]. Auch für stumme zerebrale Ischämien ist die unerkannte oder nicht ausreichend behandelte arterielle Hypertonie der wichtigste Risikofaktor. Der nächtliche Blutdruck scheint für das Auftreten von stummen Ischämien von besonderer Bedeutung zu sein [27]. Verbesserung des MMSE assoziiert und ist eine ef-
Gute Evidenz | Eine arterielle Hypertonie im mittleren Lebensalter hängt mit dem Auftreten einer Evidenz inzwischen sehr gut. Mehrere Kohorten aus verschiedenen Ländern (Schweden, Japan, Hawaii) zeigen konsistent eine statistisch signifikante Assoziation [18, 28-30]. Im Verlauf von 1020 Jahren erhöht sich das Demenzrisiko auf das 4-5-Fache [31-33]. Bereits bei „hoch-normalem“ Blutdruck konnten ungünstige Veränderungen der kognitiven Funktion in diesem Zeitraum festgestellt werden [34].

Klinisch stumme Ischämien verdoppeln das Risiko für eine Demenz über eine Beobachtungszeit von 3,5 Jahren und erhöhen das Schlaganfallrisiko [35, 36]. Demenz jeglicher Art zusammen. Dafür ist die 
Veränderung der Blut-Hirnschranke | Die arterielle Hypertonie schädigt das Gehirn nicht nur indirekt durch zerebrale Ischämien, sondern auch direkt. Sie vermindert die vaskuläre Stabilität der Blut-Hirn-Schranke, was einen Protein-Übertritt in das Hirngewebe erleichtert [37]. Hierdurch können die Zellen geschädigt werden und es kann sich Beta-Amyloid anreichern, das in der Alzheimer-Pathologie eine wesentliche Rolle spielt. In fast allen Studien sind höhere Blutdruckwerten signifikant mit einer Reduktion des Hirnvolumens assoziiert. Besonders betroffen ist der Hippocampus [38].

Risikofaktor Hypotonie? | Bei Patienten mit manifester Demenz, oder kurz davor, finden sich dagegen überwiegend niedrige Blutdruckwerte [39]. Diese Beobachtung wurde auch in der Honolulu-Asia-Aging-Study (HAAS) bestätigt [40]. Ob dies auf die Gehirnerkrankung oder einen veränderten Lebensrhythmus, Gewichtsverlust oder andere metabolische Veränderungen zurückzuführen ist, ist nicht sicher geklärt.

\section{Hypertonie und vaskuläre Demenz}

Zusammenhang belegt | Trotz methodischer Einschränkungen (Hypertoniedauer und Blutdruckhöhe nur anamnestisch erfasst oder gemessen) ist die Evidenz für einen Zusammenhang von VD und arteriellen Hypertonie sehr gut. Bei 70-jährigen Patienten, deren Blutdruckwerte über 20 Jahre dokumentiert wurden, korrelierten erhöhte Werte im Alter von 50 Jahren mit einer kognitiven Verschlechterung im Alter von 70 Jahren [41]. Bestätigt wird dies durch weitere Studien:

- Eine Meta-Analyse hat gezeigt, dass die Inzidenz der VD bei Hypertonie mit einer Odds ratio von 1,59 erhöht ist [42].

- In drei aussagekräftigen Studien an asiatischen Personen war ein Bluthochdruck im mittleren Lebensalter prospektiv mit der Inzidenz einer vaskulären Hypertonie assoziiert und erhöhte das Risiko auf das 2-3-Fache [43-45].

- In einem erweiterten Follow-up der HisayamaStudie war auch eine Hypertonie im höheren Lebensalter mit der vaskulären Demenz-Inzidenz assoziiert (OR 1,53).

\section{Hypertonie und Alzheimer-Demenz}

Assoziation nicht geklärt | Der Zusammenhang zwischen Hypertonie im mittleren Alter und dem Auftreten einer Alzheimer-Demenz (AD) ist hingegen nicht eindeutig belegt [46-48]. Im "World Alzheimer Report 2014" wird auf Schwierigkeiten bei der Beurteilung von Studien hingewiesen [46, 49, 50].

- Hierzu gehören die teils groben Blutdruckkategorien ohne Zwischenbereiche, z.B. >160 vs. $\leq 140 \mathrm{mmHg}$ und $>140$ vs. $<120 \mathrm{mmHg}[31,51]$.
- An einer chinesische Studie $(\mathrm{n}=16448)$ wurde das Alter der Patienten kritisiert: Zu Beginn der Studie waren bereits 75\% der Patienten über 60 Jahre alt. Somit konnte keine Aussage zum Blutdruck im „mittleren Lebensalter“ getroffen werden. Zudem wurden auch gemischte Demenzformen in die Auswertung aufgenommen $[52,53]$. Die Studie hatte eine Risikoerhöhung für eine Alzheimer-Demenz von 1,97 ergeben.

Problem Mischformen | Die AlzRisk-Meta-Analyse von 2011 kommt zwar zu dem Schluss, dass keine sichere Assoziation zwischen Hypertonie und der Inzidenz der Alzheimer-Erkrankung besteht doch es gibt sehr viele Patienten mit Mischformen. Aus diesem Grund wird der Anteil kognitiver Verschlechterung durch vaskuläre Ursachen bei dieser Betrachtung vermutlich unterschätzt [47].

Wenn eine Hypertonie erst im höheren Lebensalter auftritt, scheint dies die Inzidenz von AD nicht zu erhöhen [54].

Weitere sorgfältige systematische Studien, in denen auch der Einfluss einer antihypertensiven Therapie im mittleren Lebensalter untersucht wird, sind zweifellos erforderlich.

Andere Normwerte bei Hochbetagten ? I Möglicherweise gelten für sehr alte Menschen andere Normwerte. Vermutlich ist die gestörte Autoregulation des Gehirns hierfür verantwortlich. Neuere Erkenntnisse aus einer Studie an über 90-jährigen Patienten (90+-Study) haben gezeigt, dass Personen, die in der 8. Lebensdekade eine arterielle Hypertonie entwickelten, ein signifikant niedrigeres Risiko für Demenz aufwiesen (-41\%). Bei einer Manifestation ab dem 90. Lebensjahr war das Demenz-Risiko um 55\% niedriger [55].

\section{Antihypertensive Therapie}

Kein Placebo I Die Therapieeffekte zu beurteilen, bietet eine Reihe von Schwierigkeiten. Aus ethischen Gründen gibt es keine wirklich Placebokontrollierten Studien. So kommen in den Studien auch in den Placeboarmen unterschiedliche Antihypertensiva zum Einsatz. Hierdurch werden Blutdruckunterschiede zwischen den Gruppen deutlich gemindert.

Ein Cochrane Review von „Placebokontrollierten“ Studien mit 15936 Patienten ohne vorherigen Schlaganfall konnte den Einfluss einer antihypertensiven Therapie auf die Demenzrate nicht eindeutig belegen.

Allerdings war das mittlere Alter der Patienten mit 75,4 Jahren relativ hoch, sodass keine Effekte einer früh initiierten Therapie erfasst wurden [56]. 
Studienlage | Seit mehr als 30 Jahren werden in Studien zur antihypertensiven Therapie auch Einflüsse auf die kognitive Funktion bzw. das Demenzrisiko untersucht.

- Vorteile zugunsten der antihypertensiven Therapie fanden sich in der Syst-Eur-Studie unter Kalziumantagonisten-Therapie (oft in Kombination mit einem ACE-Hemmer). Bei Patienten mit isoliert systolischer Hypertonie sank die Demenzrate unter der Therapie um 50\% [57, 58].

- In der PROGRESS-Studie reduzierte eine antihypertensive Therapie mit Perindopril und Indapamid bei Patienten nach zerebralem Insult zwar nicht die Demenzrate. Jedoch sank das Risiko einer kognitiven Verschlechterung bei durchschnittlicher Blutdrucksenkung von 9/4 mmHg um $19 \%$, da Rezidiv-Schlaganfälle verhindert wurden [59].

- In der Rotterdam-Studie (>6000 Patienten) war die Einnahme von Antihypertensiva mit einer $8 \%$-igen Risikoreduktion für Demenz pro Therapiejahr für Personen $\leq 75$ Jahre assoziiert [60].

- In einer Studie mit 32-jähriger Beobachtungsdauer stieg der systolische Blutdruck bei Patienten, die im Verlauf eine Demenz entwickelten, stärker an. Dies ließ sich durch eine antihypertensive Therapie modifizieren [61].

- In der HAAS-Studie (12 Jahre Beobachtungszeit) wurde das Demenzrisiko von Patienten mit gut eingestelltem Bluthochdruck im Vergleich zu schlecht behandelten Hypertonikern um $60 \%$ abgesenkt [62].

Risikofaktor zerebrale Ischämie | Bedeutsam ist auch, stumme zerebrale Ischämien durch eine antihypertensive Therapie zu verhindern. Dies konnte von Sugiyama et al. an einer kleinen Patientenzahl mit unterschiedlicher antihypertensiver Medikation gezeigt werden [63].

Die Zielwerte der antihypertensiven Therapie nach einem Schlaganfall - auch im Hinblick auf die Kognition - werden in der derzeit noch laufenden ESH-SHOT-Studie untersucht [64].

Risikofaktor Blutdruckvariabilität | Analysen der ONTARGET-Studie zeigen, dass auch die systolische Blutdruckvariabilität die kognitiven Funktionen zu beeinflussen scheint. Antihypertensiva, die die Variabilität reduzieren, könnten somit vorteilhaft sein [65].

Kognitionsverbesserung möglich? | Ob eine Verbesserung des kognitiven Niveaus unter antihypertensiver Therapie möglich ist, ist nur in kleineren Studien untersucht worden.

- Lehrl et al. konnten in einer Studie mit 31 Patienten mit Hypertonie und leichten Hirnleistungsstörung nachweisen, dass Calcium-Antagonisten die kognitive Funktion signifikant stärker verbessern als Diuretika [66].
- Tedesco et al. haben bei 69 Patienten mit initial bestehenden Hirnleistungsstörung gezeigt, dass sich die kognitive Funktion über 2 Jahre durch Losartan verbessert. Hydrochlorothiazid erbrachte hingegen keine Veränderung [67].

Eine antihypertensive Therapie kann auch dazu beitragen, die kognitiven Situation bei $A D$ positiv zu beeinflussen. Vermutlich wird dabei die Progredienz der vaskulären Komponente verlangsamt [68].

\section{Antihypertensive Therapie bei Älteren}

Behandlung vorteilhaft | Auch bei älteren Patienten ist eine antihypertensive Therapie tendenziell mit einem geringeren Demenzrisiko assoziiert [69]. Zumindest lässt sich auch bei ihnen keine Verschlechterung kognitiver Funktionen durch antihypertensive Therapie erkennen, wenn noch keine Demenz vorliegt.

Die Evidenz für den Nutzen einer antihypertensiven Therapie ist auch bei älteren Patienten sehr gut, da generell das Risiko für kardiovaskuläre Morbidität und Mortalität reduziert wird.

Profit auch bei Einschränkungen? | Die Frage, ob auch Patienten mit bestehenden kognitiven Einschränkungen oder einer Demenz von einer antihypertensiven Therapie profitieren, ist nicht sicher geklärt [70]. Studien kommen zu unterschiedlichen Ergebnissen, jedoch ist die Aussagekraft wegen kleiner Patientenzahlen und methodischer Schwierigkeiten eingeschränkt [71]. Interessante Daten liefert eine italienische Studie mit 172 älteren Patienten (im Mittel 79 Jahre) mit Demenz (68\%) oder leichter kognitiver Einschränkung (32\%) und einer Beobachtungsdauer von 9 Monaten [17]:

- Bei Patienten im niedrigsten Terzil des systolischen ABDM-Tagesblutdrucks $(\leq 128 \mathrm{mmHg}$ ) fiel der MMST-Score stärker ab als bei Patienten im mittleren (129-144 mmHg) und höchsten Terzil ( $\geq 145 \mathrm{mmHg}$ ). Der Zusammenhang bestand nur im Rahmen einer antihypertensiven Therapie. Eine zu strenge Blutdruckeinstellung wäre demnach von Nachteil.

- Zudem war die ABDM zuverlässiger als die Praxisblutdruckmessung. Daher sollte die ABDM die Basis einer Therapieentscheidung sein.

Eine weitere Studie hat gezeigt, dass sich die kognitive Funktion bei Alzheimer-Patienten möglicherweise verbessert, wenn sie mit ACE-Hemmern behandelt werden, die die Blut-HirnSchranke passieren können [72]. Hierfür bedarf es jedoch weiterer klinischer Studien. 
Einige Autoren kommen zu dem Schluss, dass nach gegenwärtiger Datenlage bevorzugt Calcium-Antagonisten oder ACE-Hemmer bei älteren Patienten mit Demenz eingesetzt werden sollten [73].

\section{Welche Antihypertensiva sind besonders geeignet?}

Große Langzeitstudien entscheidend I Um substanzklassenspezifische Effekte zu beurteilen, sind große Patientenzahlen und lange Beobachtungsdauern erforderlich.

Die Frage, ob nur bestimmte antihypertensive Substanzen in der Lage sind, kognitive Funktionsstörungen und Demenz zu verhindern, lässt sich derzeit nicht abschließend beantworten.

Tierexperimentelle Studien | Untersuchungen an Mäusen haben gezeigt, dass eine kontinuierliche Aktivierung des Renin-Angiotensin-Systems die kognitiven Funktionen beeinträchtigt.

- Dies kann durch eine Therapie mit einem AT1Rezeptorblocker (ARB) verhindert werden. Hydralazin hingegen war dazu nicht in der Lage trotz gleicher Blutdrucksenkung [74].

- Weitere Untersuchungen beschäftigen sich mit der Rolle des AT4-Rezeptors [75]. Es wurde belegt, dass sich die kognitive Funktion durch Angiotensin-(1-7) bei chronischer zerebraler Minderperfusion verbessert [76]. Ob dies auch bei Menschen gilt, ist derzeit offen.

ACE-Hemmer | In der Cardiovascular-Health-Study (Cognition-Substudy) verringerten ACE-Hemmer, die die Blut-Hirnschranke passieren können, in einem Zeitraum von 6 Jahren den Punktwertabfall in einem modifizierten MMSE um 65\%. Patienten, die keine zentral aktiven ACE-Hemmer erhielten, hatten hingegen ein höheres Demenzrisiko [77].

AT1-Rezeptorblocker I In einer bevölkerungsbasierten Kohortenstudie (Taiwan-NationalHealth-Insurance-Research-Database) wurden 24531 passende Paare (1:1) mit bzw. ohne ARBBehandlung gebildet. Die Beobachtungsphase betrug 12 Jahre.

- Bei 1322 Patienten (5.4\%) der ARB-Gruppe wurde eine Demenz festgestellt. In der Vergleichsgruppe ohne ARB war dies bei $2181 \mathrm{~Pa}-$ tienten (8.9\%) der Fall.

- Das Risiko für eine Demenz unter ARB betrug für AD 0,53 und für VD 0,63.

- Patienten mit höheren kumulativen ARB-Dosen (>1460 definierte Tagesdosen) hatten ein geringeres Risiko als Patienten mit niedrigen Dosen (Hazard ratio 0,37 vs. 0,61; $\mathrm{p}<0.05$ ) [78].

Auch andere Autoren kommen zu dem

Schluss, daß ARB derzeit als besonders

effektiv angesehen werden können [79-81].

Betablocker | Andererseits wird in einer Auswertung der HAAS-Studie bei 2197 älteren Männern mit Hypertonie (Durchschnittsalter 77 Jahre bei Beobachtungsbeginn) ein Vorteil von Betablockern gegenüber anderen Antihypertensiva berichtet. Während der medianen Nachbeobachtungszeit von 5,8 Jahren entwickelten 854 Männer eine kognitive Einschränkung. Die Einnahme von Betablockern als einzige antihypertensive Therapie zu Studienbeginn war mit einem niedrigen Risiko für kognitive Einschränkungen assoziiert [82].

Konsequenz für Klinik und Praxis

- Es besteht eine sehr gute Evidenz, dass eine arterielle Hypertonie im mittleren Lebensalter mit dem höheren Demenzrisiko im späteren Leben assoziiert ist vornehmlich bedingt durch die VD.

- Eine sorgfältige Diagnostik und konsequente antihypertensive Therapie im mittleren Lebensalter sind daher von entscheidender Bedeutung, um das Demenzrisiko zu reduzieren.

- Die Einstellung von normotonen Blutdruckwerten ist ausschlaggebend, um kognitive Funktionsstörungen und Hirninfarkte (stumm oder manifest) zu verhindern.

- Das ambulante Blutdruck-Monitoring ist unerlässlich in der Blutdruckdiagnostik und Therapiekontrolle.

- Bislang gibt es keine medikamentöse Therapie, um eine manifeste VD zurückzubilden. Werden alle Risikofaktoren konsequent behandelt, kann eine gewisse Verbesserung der kognitiven Fähigkeiten erreicht werden.

- Eine gesunde Lebensführung mit regelmäBiger körperlicher und geistiger Aktivität, Pflege sozialer Kontakte sowie mäßiger und ausgewogener Ernährung sind die Basis der Demenzprävention.

\section{Literatur}

1 Deutsches Institut für Medizinische Information und Dokumentation (DIMDI). Internationale statistische Klassifikation der Krankheiten und verwandter Gesundheitsprobleme. 10. Revision German Modification Version 2015. https://www.dimdi.de/static/de/klassi/icd-10-gm/ kodesuche/onlinefassungen/htmlgm2015/ (letzter Zugriff: 7.9.2015)

Vollständiges Literaturverzeichnis unter http://dx.doi.org/10.1055/s-0041-105598

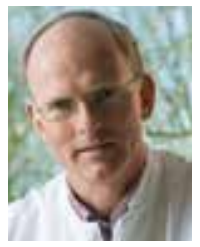

PD Dr. med. Stephan Lüders

ist leitender Oberarzt in der Abteilung für Innere Medizin-Geriatrie am St. Josefs-Hospital, Cloppenburg.

stephan.lueders@kk-om.de

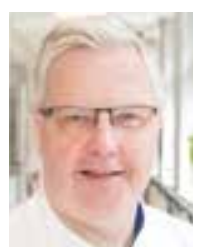

Prof. Dr. med. Joachim Schrader ist Ärztlicher Direktor und Chefarzt der Abteilung für Allgemeine Innere MedizinNephrologie und Geriatrie am St. Josefs-Hospital, Cloppenburg. joachim.schrader@kk-om.de

Interessenkonflikt Die Autoren geben an, dass kein Interessenskonflikt besteht.

DOI 10.1055/s-0041-105598

Dtsch Med Wochenschr 2015; 140: 1599-1603 (c) Georg Thieme Verlag KG . Stuttgart · New York . ISSN 0012-0472 
2 Gauthier S, Reisberg B, Zaudig M et al. Mild cognitive impairment. Lancet 2006; 367: 1262-1270

3 Hachinski V, ladecola C, Petersen RC et al. National Institute of Neurological Disorders and StrokeCanadian Stroke Network vascular cognitive impairment harmonization standards. Stroke 2006; 37: 2220-2241

4 Van Straaten ECW, Scheltens P, Knol DL et al. Operational definitions for the NINDS-AIREN criteria for vascular dementia: an interobserver study. Stroke 2003; 34: 1907-1912

5 Schneider JA, Arvanitakis Z, Bang W et al. Mixed brain pathologies account for most dementia cases in community-dwelling older persons. Neurology 2007; 69: 2197-2204

6 Gutzmann H, Qazi A. Depression associated with dementia. Z Gerontol Geriat 2015; 48: 305-311

7 Kalbe E, Calabrese P, Kohn $\mathrm{N}$ et al. Screening for cognitive deficits in Parkinson's disease with the Parkinson neuropsychometric dementia assessment (PANDA) instrument. Parkinsonism Relat Disord 2008; 14: 93-101

8 Frisoni GB, Bocchetta M, Chételat G et al. Imaging markers for Alzheimer disease: Which vs how. Neurology 2013; 81(5): 487-500

9 Savva GM, Wharton SB, Ince PG et al. Age, neuropathology, and dementia. N Engl ] Med 2009; 360: 2302-2309

10 DGN. Leitlinien für Diagnostik und Therapie in der Neurologie: Diagnose und Therapie von Demenzen (2009).

http://www.dgn.org/images/red_leitlinien/ LL_2012/pdf/II_15_2012_diagnose_und_therapie_von_demenzen.pdf (letzter Zugriff: 7.9. 2015)

11 Kessler ], Calabrese P, Kalbe E et al. DemTect: A new screening method to support diagnosis of dementia. Psycho 2000; 26: 343-347

12 Takata Y, Ansai T, Soh I et al. Cognitive function and 10 year mortality in an 85 year-old communitydwelling population. Clin Interv Aging 2014; 9: 1691-1699

13 Wiederkehr S, Simard M, Fortin C et al. Validity of the clinical critera for vascular dementia: A critical review. Part II. J Neuropsychiatry Clin Neurosci 2008; 20: 162-177

14 DGN. Leitlinien für Diagnostik und Therapie in der Neurologie: Diagnostik und Therapie von Gedächtnisstörungen Entwicklungsstufe (2012) http://www.awmf.org/uploads/tx_szleitlinien/030124I_S2e_Ged\%C3\%A4chtnisst\%C3\%B6rungen_ Diagnostik_Therapie_2012-verl\%C3\%A4ngert.pdf (letzter Zugriff: 7.9.2015)

15 Lüders S, Schrader J, Zidek W et al. Ambulatory blood pressure measurement in prehypertension: Analysis of the PHARAO-Study: Prevention of Hypertension with the ACE-inhibitor Ramipril in patients with high-normal blood pressure - a prospective, randomised, controlled prevention trial of the German Hypertension League. J Hypertension 2008, 26: 1487-1496

16 Lüders S, Dechend R, Eckert S et al. 24-h-Langzeitblutdruckmessung (ABDM). Der Kardiologe 2013; 7: 194-208

17 Mossello E, Pieraccioli M, Nesti $\mathrm{N}$ et al. Effects of low blood pressure in cognitively impaired elderly patients treated with antihypertensive drugs. JAMA Intern Med 2015; 175: 578-585

18 Rönnemaa E, Zethelius B, Lannfelt L et al. Vascular risk factors and dementia: 40-year follow-up of a population-based cohort. Dement Geriatr Cogn Disord 2011; 31: 460-466
19 Lüders S, Saathoff U, Haller H et al. Häufigkeit kognitiver Funktionsstörungen bei Patienten mit Begleiterkrankungen - Ergebnisse des DemTectRegisters der Schlaganfall-Kommission der Deutschen Hypertonie-Gesellschaft. Berlin: DHL-Kongress; 2012

20 Zieschang T, Hauer K, Schwenk M. Körperliches Training bei Menschen mit Demenz. Dtsch Med Wochenschr 2012; 1370: 1552-1555

21 Hess NCL, Dieberg G, McFarlane JR et al. The effect of exercise intervention on cognitive performance in persons at risk of, or with, dementia: $A$ systematic review and meta-analysis. Healthy Aging Research 2014; 3: 3

22 Brayne C, Ince PG, Keage HA et al. Education, the brain and dementia: neuroprotection or compensation. Brain 2010; 133: 2210-2216

23 Leys D, Henon H, Mackowiak-Cordoliani MA et al. Poststroke dementia. Lancet Neurol 2005; 4: 752-759

24 Mellon L, Brewer L, Hall P et al. Cognitive impairment six months after ischaemic stroke: a profile from the ASPIRE-S study. BMC Neurol 2015; 15: 31

25 Dufouil C, Godin O, Chalmers J et al. Cerebral white matter hyperintensities predict severe cognitive decline in patients with cerebrovascular disease history. Stroke 2009; 40: 2219-2221

26 Sierra C, De la Sierra A, Mercader J et al. Silent cerebral white matter lesions in middle-aged essential hypertensive patients. J Hypertens 2002; 20: 519-524

27 Watanabe N, Imai Y, Nagai K et al. Nocturnal blood pressure and silent cerebrovascular lesions in elderly Japanese. Stroke 1996; 27: 1319-1327

28 Launer LJ, Ross GW, Petrovitch $\mathrm{H}$ et al. Midlife blood pressure and dementia: the Honolulu-Asia aging study. Neurobiol Aging 2000; 21: 49-55

29 Freitag MH, Peila R, Masaki K et al. Midlife pulse pressure and incidence of dementia: the HonoluluAsia Aging Study. Stroke 2006; 37: 33-37

30 Kivipelto M, Helkala EL, Laakso MP et al. Apolipoprotein E epsilon4 allele, elevated midlife total cholesterol level, and high midlife systolic blood pressure are independent risk factors for late-life Alzheimer disease. Ann Intern Med 2002; 137 : 149-155

31 Launer LI, Masaki K, Petrovitch $\mathrm{H}$ et al. The association between midlife blood pressure levels and late-life cognitive function. JAMA 1995; 274: 1846-1851

32 Morris MC, Scherr PA, Hebert LE et al. Association of incident Alzheimer's disease and blood pressure measured from 13 years before to 2 years after diagnosis in a large community study. Arch Neurol 2001; 58: 1640-1646

33 Qiu C, Winblad B, Fratiglioni L. The age-dependent relation of blood pressure to cognitive function and dementia. Lancet Neurol 2005; 4: 487-499

34 Knecht S, Wersching $\mathrm{H}$, Lohmann $\mathrm{H}$ et al. High-normal blood pressure is associated with poor cognitive performance. Hypertension 2008; 51: 663-668

35 Vermeer SE, Prins ND, Heijer den T et al. Silent brain infarcts and the risk of fementia and cognitive decline. N Engl ] Med 2003; 348: 1215-1222

36 Bernick C, Kuller L, Dulberg C et al. Silent MRI infarcts and the risk of future stroke: The cardiovascular health study. Neurology 2001; 57: 1222-1229

37 Kalaria RN. Vascular basis for brain degeneration: faltering controls and risk factors for dementia. Nutr Rev 2010; 68: S74-87 
38 Beauchet O, Celle S, Roche F et al. Blood pressure levels and brain volume reduction: a systematic review and meta-analysis. J Hypertens 2013; 31: 1502-1516

39 Skoog I, Lernfelt B, Landahl S et al. A 15-year longitudinal study of blood pressure and dementia. Lancet 1996; 347: 1141-1145

40 Stewart R, Xue QL, Masaki K et al. Change in blood pressure and incident dementia: a 32-year prospective study. Hypertension 2009; 54: 233-240

41 Kilander L, Nyman $\mathrm{H}$, Boberg $\mathrm{M}$ et al. Hypertension is related to cognitive impairment: A 20-year follow-up of 999 men. Hypertension 1998; 31: 780-786

42 Sharp SI, Aarsland D, Day S et al. Hypertension is a potential risk factor for vascular dementia: systematic review. Int ] Geriatr Psychiatry 2011; 26: 661-669

43 Ross GW, Petrovitch H, White LR et al. Characterization of risk factors for vascular dementia: the Honolulu-Asia Aging Study. Neurology 1999; 53 : 337-343

44 Ueda K, Kawano H, Hasuo Y et al. Prevalence and etiology of dementia in a Japanese community. Stroke 1992; 23: 798-803

45 Yoshitake T, Kiyohara Y, Kato I et al. Incidence and risk factors of vascular dementia and Alzheimer's disease in a defined elderly Japanese population: the Hisayama Study. Neurology 1995; 45: 1161-1168

46 Prince M, Albanese E, Guerchet M. World Alzheimer Report 2014. Dementia and risk reduction. An analysis of protective and modifiable factors. London: Alzheimer's Disease International (ADI); 2014

47 Power MC, Weuve J, Gagne J] et al. The association between blood pressure and incident Alzheimer disease: a systematic review and meta-analysis. Epidemiology 2011; 22: 646-659

48 Meng XF, Yu JT, Wang HF et al. Midlife vascular risk factors and the risk of Alzheimer's disease: a systematic review and meta-analysis. J Alzheimers Dis 2014; 42: 1295-1310

49 Barnes DE, Yaffe K. The projected effect of risk factor reduction on Alzheimer's disease prevalence. Lancet Neurol 2011; 10: 819-828

50 Norton S, Matthews FE, Barnes DE et al. Potential for primary prevention of Alzheimer's disease: an analysis of population-based data. Lancet Neurol 2014; 13: 788-794

51 Kivipelto M, Helkala EL, Laakso MP et al. Midlife vascular risk factors and Alzheimer's disease in later life: longitudinal, population based study. BM] 2001; 322: 1447-1451

52 Wu C, Zhou D, Wen C et al. Relationship between blood pressure and Alzheimer's disease in Linxian County, China. Life Sci 2003; 72: 1125-1133

53 Whitmer RA, Sidney S, Selby J et al. Midlife cardiovascular risk factors and risk of dementia in late life. Neurology 2005; 64: 277-281

54 Ninomiya T, Ohara T, Hirakawa Y et al. Midlife and late-life blood pressure and dementia in Japanese elderly: the Hisayama study. Hypertension 2011; 58: 22-28

55 Corrada M, Hayden KM, Bullain SS et al. Age of onset of hypertension and risk of dementia in the oldest-old: The 90+ Study. Alzheimers Dement 2014; 10: P501

56 McGuinness B, Todd S, Passmore P et al. Blood pressure lowering in patients without prior cerebrovascular disease for prevention of cognitive impairment and dementia. Cochrane Database Syst Rev 2009; 4: CD004034
57 Forette F, Seux M-L, Staessen JA et al. Prevention of dementia in randomised double-blind placebocontrolled Systolic Hypertension in Europe (Syst-Eur) trial. Lancet 1998; 352: 1347-1351

58 Forette F, Seux ML, Staessen JA et al. The prevention of dementia with antihypertensive treatment. New evidence from the systolic hypertension in Europe (Syst-Eur) Study. Arch Int Med 2002; 162: 2046-2052

59 Tzourio C, Anderson C, Chapman N et al. Effects of blood pressure lowering with perindopril and indapamide therapy on dementia and cognitive decline in patients with cerebrovascular disease. Arch Intern Med 2003; 163: 1069-1075

60 Haag MDM, Hofman A, Koudstaal PJ et al. Duration of antihypertensive drug use and risk of dementia. A prospective cohort study. Neurology 2009; 72: 1727-1734

61 Stewart R, Xue QL, Masaki K et al. Change in blood pressure and incident dementia a 32-year prospective study. Hypertension 2009; 54 233-240

62 Peila R, White LR, Masaki K et al. Reducing the risk of dementia. Efficacy of long-term treatment of hypertension. Stroke 2006; 37: 1165-1170

63 Sugiyama T, Lee JD, Shimizu H et al. Influence of treated blood pressure on progression of silent cerebral infarction. J Hypertens 1999; 17: 679-684

64 Zanchetti A, Liu L, Mancia Get al. Blood pressure and LDL-cholesterol targets for prevention of recurrent strokes and cognitive decline in the hypertensive patient: design of the European Society of Hypertension - Chinese Hypertension League Stroke in Hypertension Optimal Treatment randomized trial. J Hypertens 2014; 32: 1888 1897

65 Böhm M, Schumacher H, Leong D et al. Systolic blood pressure variation and mean heart rate is associated withcognitive dysfunction in patients with high cardiovascular risk. Hypertension 2015; 65: 651-661

66 Lehrl S, Gräßel E, Eicke C. Wirkung von Felodipin bei hypertonen Patienten mit leichten Hirnleistungsstörungen in einer randomisierten Doppelblindstudie. Dtsch med Wochenschr 2000; 125: 1350-1356

67 Tedesco MA, Ratti G, Mennella S et al. Comparison of losartan and hydrochlorothiazide on cognitive function and quality of life in hypertensive patients. Am J Hypertension 1999; 12: 1130-1134

68 Duron E, Rigaud AS, Dubail D et al. Effects of Antihypertensive Therapy on Cognitive Decline in Alzheimer's Disease. Am J Hypertens 2009; 22: 1020-1024

69 Peters R, Beckett N, Forette F et al., Incident dementia and blood pressure lowering in the Hypertension in the Very Elderly Trial cognitive function assessment (HYVET-COG): a doubleblind, placebo controlled trial. Lancet Neurol 2008; 7: 683-689

70 Van der Wardt V, Logan P, Conroy S et al. Antihypertensive treatment in people with dementia. J Am Med Dir Assoc 2014; 15: 620-629

71 Beishon LC, Harrison JK, Harwood RH et al. The evidence for treating hypertension in older people with dementia: a systematic review. J Hum Hypertens 2014; 28: 283-287

72 Ohrui T, Tomita N, Sato-Nakagawa T et al. Effects of brain-penetrating ACE inhibitors on Alzheimer disease progression. Neurology 2004; 63: 1324-1325

73 Kaiser EA, Lotze U, Schäfer HH. Increasing complexity: which drug class to choose for treatment of hypertension in the elderly? Clin Interven Aging 2014; 9: 459-475 
74 Inaba S, Iwai M, Furuno M et al. Continuous activation of renin-angiotensin system impairs cognitive function in renin / angiotensinogen transgenic mice. Hypertension 2009; 53: 356

75 Wright JW, Harding JW. The angiotensin AT4 receptor subtype as a target for the treatment of memory dysfunction associated with Alzheimer's disease. JRAAS 2008; 9: 226-237

76 Xie W, Zhu D, Ji L et al., Angiotensin-(1-7) improves cognitive function in rats with chronic cerebral hypoperfusion. Brain Res 2014; 1573: 44-53

77 Sink KM, Leng X, Williamson J et al. Angiotensinconverting enzyme inhibitors and cognitive decline in older adults with hypertension. Arch Intern Med. 2009; 169: 1195-1202

78 Chiu WC, Ho WC, Lin MH et al., Angiotension receptor blockers reduce the risk of dementia. J Hypertens 2014; 32: 938-947

79 Fogari R, Preti P, Mugellini A et al. Influence of losartan and atenolol on cognitive function in very elderly hypertensive patients. Am J Hypertens 2002; 15: $36 \mathrm{~A}$

80 Levi Marpillat N, Macquin-Mavier I, Tropeano Al et al. Antihypertensive classes, cognitive decline and incidence of dementia: a network meta-analysis. J Hypertens 2013; 31: 1073-1082

81 Schrader J, Lüders S, Kulschewski A et al. Morbidity and mortality after stroke, Eprosartan compared with Nitrendipine for secondary prevention: principal results of a prospective randomized controlled study (MOSES). Stroke 2005; 36: 1218-1226

82 Gelber RP, Ross GW, Petrovich H et al. Antihypertensive medication use and risk of cognitive impairment: The Honolulu-Asia Aging Study. Neurology 2013; 81: 888-895 